\title{
Optogenetic defibrillation terminates ventricular arrhythmia in mouse hearts and human simulations
}

\author{
Tobias Bruegmann, ${ }^{1,2}$ Patrick M. Boyle, ${ }^{3}$ Christoph C. Vogt, ${ }^{1}$ Thomas V. Karathanos, ${ }^{3}$ Hermenegild J. Arevalo, ${ }^{3}$ \\ Bernd K. Fleischmann, ${ }^{1}$ Natalia A. Trayanova, ${ }^{3}$ and Philipp Sasse ${ }^{1}$ \\ IInstitute of Physiology I, Life and Brain Center, and 'Research Training Group 1873, University of Bonn, Bonn, Germany. ${ }^{3}$ Institute for Computational Medicine and Department of Biomedical Engineering, \\ Johns Hopkins University, Baltimore, Maryland, USA.
}

\begin{abstract}
Ventricular arrhythmias are among the most severe complications of heart disease and can result in sudden cardiac death. Patients at risk currently receive implantable defibrillators that deliver electrical shocks to terminate arrhythmias on demand. However, strong electrical shocks can damage the heart and cause severe pain. Therefore, we have tested optogenetic defibrillation using expression of the light-sensitive channel channelrhodopsin-2 (ChR2) in cardiac tissue. Epicardial illumination effectively terminated ventricular arrhythmias in hearts from transgenic mice and from WT mice after adeno-associated virus-based gene transfer of ChR2. We also explored optogenetic defibrillation for human hearts, taking advantage of a recently developed, clinically validated in silico approach for simulating infarct-related ventricular tachycardia (VT). Our analysis revealed that illumination with red light effectively terminates VT in diseased, ChR2-expressing human hearts. Mechanistically, we determined that the observed VT termination is due to ChR2-mediated transmural depolarization of the myocardium, which causes a block of voltage-dependent $\mathrm{Na}^{+}$channels throughout the myocardial wall and interrupts wavefront propagation into illuminated tissue. Thus, our results demonstrate that optogenetic defibrillation is highly effective in the mouse heart and could potentially be translated into humans to achieve nondamaging and pain-free termination of ventricular arrhythmia.
\end{abstract}

\section{Introduction}

Ventricular tachycardia (VT) and ventricular fibrillation (VF) are malignant arrhythmias, which are characterized by fast and uncoordinated electrical excitations of the myocardium, resulting in pump failure and leading to sudden cardiac death. The current state-of-the-art therapy for these life-threatening disorders is defibrillation by the delivery of high-energy electrical shocks. Patients at risk for developing ventricular arrhythmia receive implantable automatic defibrillators. Clinical studies have demonstrated the life-saving potential of this therapeutic approach; however, it is also associated with significant adverse effects, such as structural damage of cardiac tissue worsening the underlying cardiac disease, severe pain due to nonselective excitation of nerves and muscles with subsequent psychological trauma, and increased mortality (1-4). Thus, there is a need for alternative approaches that enable pain-free and less deleterious termination of lethal arrhythmia.

Optogenetics is an emerging technology that enables modulation of electrical function with light in cells overexpressing light-sensitive proteins. The light-activated cation channel channelrhodopsin-2 (ChR2) has been used to directly pace the heart with brief (1-10 ms) blue light pulses in vivo (5-8). However, these results do not imply the feasibility of optogenetic defibrillation, because cardiac pacing entails only a brief depolarization

Authorship note: T. Bruegmann and P.M. Boyle contributed equally to this work. Conflict of interest: The authors have declared that no conflict of interest exists. Submitted: June 3, 2016; Accepted: August 4, 2016.

Reference information: / Clin Invest. 2016;126(10):3894-3904. doi:10.1172/JCl88950. in a few cardiomyocytes with subsequent intrinsic excitation of the whole heart through gap-junctional electrical coupling. In contrast, defibrillation requires the simultaneous depolarization of a large mass of ventricular tissue (9), and therefore the energy required for a defibrillating shock is dramatically larger than that of a typical pacing stimulus. Optogenetics enables the selective depolarization of ChR2-expressing cardiomyocytes only, making it an attractive pain-free alternative to electrical shocks. An additional strength over electrical shocks is that optogenetics allows uniform and sustained depolarization by continuous illumination. Although this advantage has been exploited in vitro to achieve fine-grain control of excitation waves in cardiomyocyte monolayers, including reentry destabilization (10), slowing of conduction (11), and suppression of electrical activity (5), the feasibility of optogenetic defibrillation in intact hearts has never been demonstrated experimentally.

Herein, we report a new protocol to induce sustained ventricular arrhythmia in mouse hearts and provide experimental evidence that optogenetic defibrillation by illumination of the epicardial surface is a highly reliable approach. In addition, we investigate the translational potential of optogenetic defibrillation by epicardial illumination with a novel in silico approach using a model of the heart of a patient with a propensity for infarct-related VT.

\section{Results}

Optogenetic defibrillation via epicardial illumination in intact hearts of transgenic mice. First, we explored optogenetic defibrillation via epicardial illumination in hearts of transgenic mice expressing ChR2 in all cardiomyocytes (5). Because of the small size of the 
A

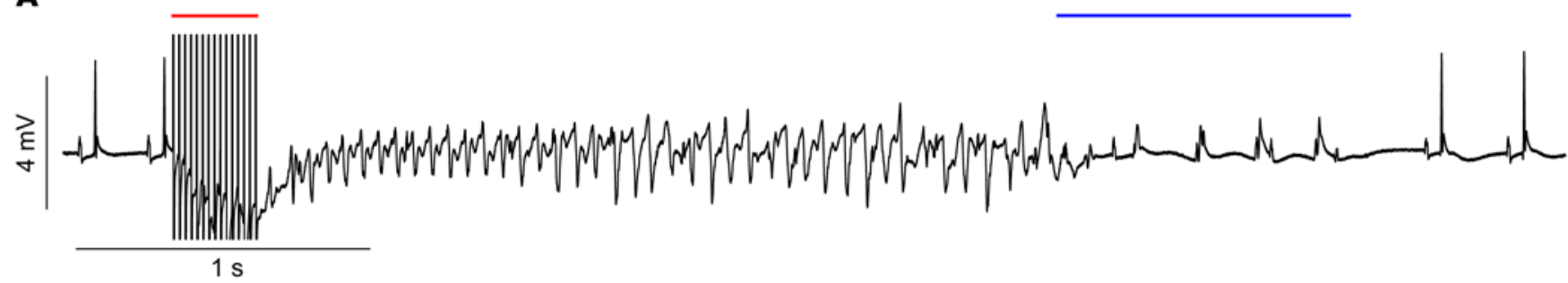

B

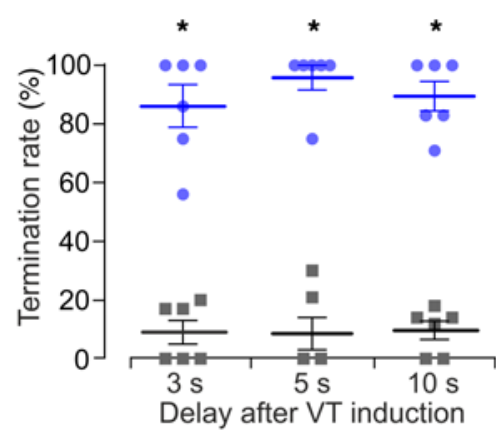

D

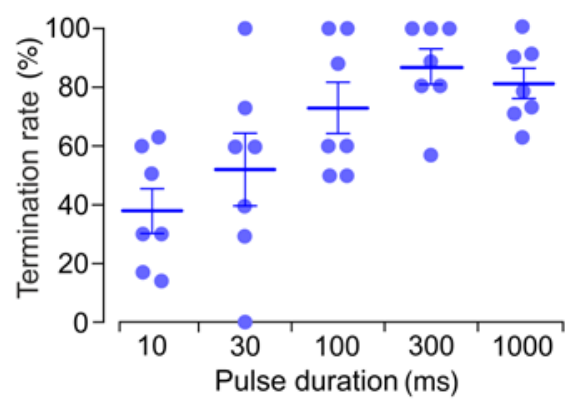

ChR2 $0.4 \mathrm{~mW} / \mathrm{mm}^{2}$ ChR2 no light

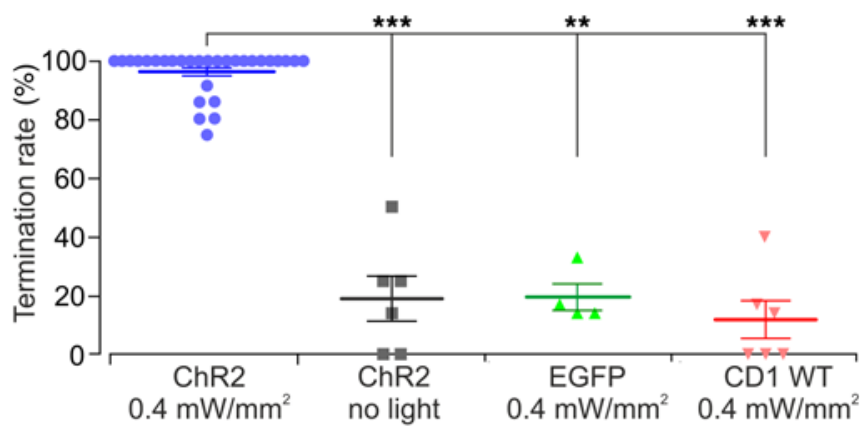

E

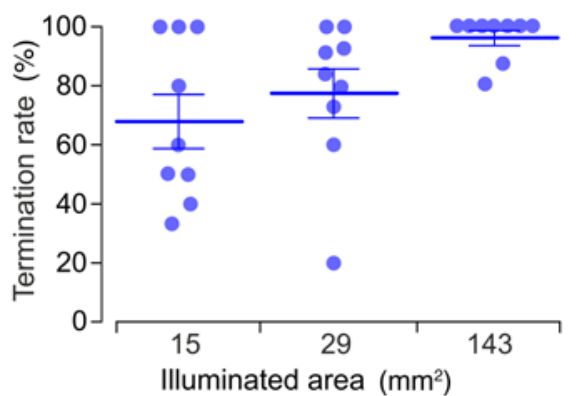

$\mathbf{F}$

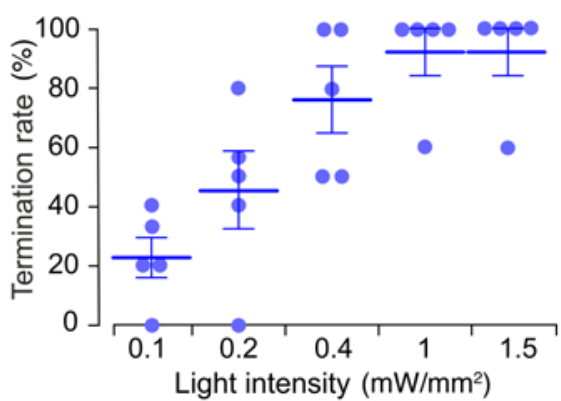

Figure 1. Optogenetic defibrillation in transgenic hearts. (A) Representative ECG (black) from an explanted heart expressing ChR2. Sustained VT was induced by electrical burst stimulation $\left(50 \mathrm{~Hz}\right.$, red bar) and terminated by epicardial illumination with blue light (blue bar, $470 \mathrm{~nm}, 1 \mathrm{~second}, 0.4 \mathrm{~mW} / \mathrm{mm}^{2}$, $\left.143 \mathrm{~mm}^{2}, n=36\right)$. (B) Arrhythmia termination rate in ChR2 hearts after one 1-second-long light pulse $\left(0.4 \mathrm{~mW} / \mathrm{mm}^{2}, 143 \mathrm{~mm} \mathrm{~m}^{2}\right.$, blue) applied 3, 5, and 10 seconds after induction of arrhythmia compared with control conditions (i.e., no illumination, black) in the same time window (see Supplemental Figure 1B for experimental protocol; Wilcoxon matched pairs test, $P<0.04, n=6$ ). (C) Arrhythmia termination rate in response to a 4-light-pulse protocol (Supplemental Figure 1C) in ChR2 mouse hearts with illumination (1 second, $0.4 \mathrm{~mW} / \mathrm{mm}^{2}, 143 \mathrm{~mm}^{2}$, blue) and without illumination (black), as well as in hearts expressing only EGFP (green) and in CD1 WT hearts (red) with illumination (Kruskal-Wallis test, $P<0.0001$, with Dunn's multiple-comparison post-test; ChR2 light: $n=$ 29; ChR2 no light: $n=6$; EGFP: $n=4$; CD1 WT: $n=6$ ). (D-F) Influence of illumination duration $\left(\mathbf{D}, 0.4 \mathrm{~mW} / \mathrm{mm}^{2}, 29 \mathrm{~mm}^{2}, n=7, P=0.0007\right)$, area $(\mathbf{E}, 1 \mathrm{~second}$, $\left.0.4 \mathrm{~mW} / \mathrm{mm}^{2}, n=9, P=0.031\right)$, and light intensity $\left(\mathbf{F}, 1\right.$ second, $15 \mathrm{~mm}^{2}, n=5, P=0.013$ ) on arrhythmia termination rate (Friedman test). Each point shows the percentage of successful optogenetic defibrillation attempts in 1 heart. Data are shown as the mean $\pm \mathrm{SEM} .{ }^{*} P \leq 0.05,{ }^{* *} P \leq 0.01$, and ${ }^{* * *} P \leq 0.001$.

mouse heart, electrically induced ventricular arrhythmias in vivo are often self-terminating after several cycles (12), rendering the analysis of defibrillation efficacy impossible. Therefore, we developed a novel protocol and obtained sustained ventricular arrhythmias evoked by electrical burst stimulation or S1-S2 protocols from the base of the right ventricular wall at a point with minimal effective refractory period (ERP) in hearts perfused ex vivo with low- $\mathrm{K}^{+}(2 \mathrm{mM})$ Tyrode's solution and the $\mathrm{K}_{\mathrm{ATP}}$ channel activator pinacidil $(100 \mu \mathrm{M}$; Supplemental Figure 1A; supplemental material available online with this article; doi:10.1172/JCI88950DS1). Termination of ventricular arrhythmia was achieved by illumination of the anteroseptal epicardium with blue light $(470 \mathrm{~nm})$ in all hearts tested ( $n=36$; Figure $1 \mathrm{~A})$. These results reveal that uniform light-induced depolarization is sufficient for cardiac defibrillation. Quantitatively, application of 1 single light pulse (1 second, $0.4 \mathrm{~mW} / \mathrm{mm}^{2}, 143 \mathrm{~mm}^{2}$; Supplemental Figure 1B) resulted in arrhythmia termination with an average success rate of about $85 \%$, independent of the preceding arrhythmia duration $(3,5$, or 10 seconds; Figure 1B, blue). Control experiments without illumination showed that the rate of spontaneous arrhythmia termination in the same time window was very low $(<15 \%, P<0.04$; Figure 1B, black). To further enhance optogenetic defibrillation efficacy, we developed an illumination protocol involving 4 consecutive light pulses ( 1 second, $0.4 \mathrm{~mW} / \mathrm{mm}^{2}, 143 \mathrm{~mm}^{2}$; Supplemental Figure 1C), which yielded a very high success rate of $97 \% \pm 0.01 \%$ $(n=29$; Figure 1C, blue). Importantly, when the identical illumination protocol was applied to hearts without ChR2 expression from EGFP-expressing or CD1 WT mice, the arrhythmia termination rate in the same time window was approximately $20 \%$ (Figure 1C, green and red). This value is similar to the spontaneous termina- 
A

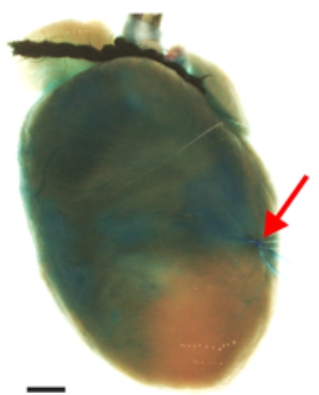

B

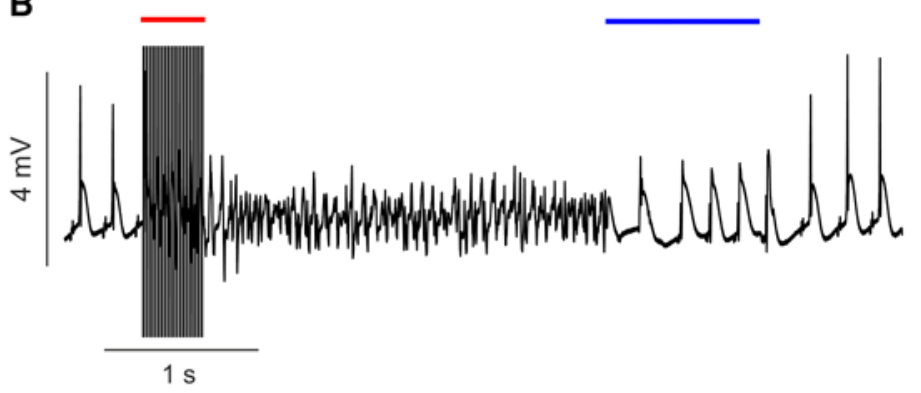

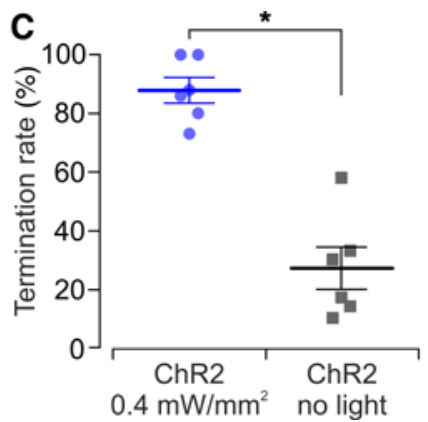

Figure 2. Optogenetic defibrillation of infarct-related ventricular arrhythmia in ChR2-expressing hearts. (A) Image of the whole heart after ligation of the left anterior descending coronary artery (red arrow) and dye perfusion (blue), highlighting the nonperfused infarct area. (B) Representative ECC (black) after ligation. Note the prominent elevation of the ST segment during normal sinus rhythm. Ventricular arrhythmia was induced by electrical burst stimulation ( $50 \mathrm{~Hz}$, red bar) and terminated by epicardial illumination with blue light (blue bar, $470 \mathrm{~nm}, 1$ second, $0.4 \mathrm{~mW} / \mathrm{mm}^{2}, 143 \mathrm{~mm} \mathrm{~m}^{2}, n=10$ ). (C) Arrhythmia termination rate in infarcted hearts induced by one 1-second-long light pulse $\left(0.4 \mathrm{~mW} / \mathrm{mm}^{2}, 143 \mathrm{~mm}^{2}\right.$, blue) applied 5 seconds after induction of arrhythmia compared with control (i.e., no illumination, black) within the same time window (see Supplemental Figure 1B for experimental protocol; Wilcoxon matched pairs test, $P=0.031, n=6$ ). Scale bar: $1 \mathrm{~mm}$. Data are shown as the mean $\pm \mathrm{SEM}$. ${ }^{*} P \leq 0.05$.

tion rate in the absence of illumination in transgenic ChR2 hearts (Figure 1C, black). Thus, neither illumination of ChR2-negative hearts nor ChR2 expression in the absence of illumination caused or facilitated arrhythmia termination.

To determine how different illumination parameters affect defibrillation success rates, sequential protocols with light stimuli of varying duration, intensity, and size of illuminated area were tested (Supplemental Figure 1, A and C). This revealed a significant influence of light stimulus duration, and the defibrillation rate was low for durations less than $100 \mathrm{~ms}$ (Figure 1D), suggesting that illumination lasting for at least the duration of the entire VT cycle $(\sim 80 \mathrm{~ms})$ was required for successful arrhythmia termination. Decreasing the illuminated area to $15 \mathrm{~mm}^{2}$ also reduced efficacy (Figure 1E), and in this situation the light intensity needed to effectively defibrillate increased from 0.4 to $1 \mathrm{~mW} / \mathrm{mm}^{2}$ (Figure $1 \mathrm{~F})$. This suggests that optogenetic depolarization of a critical portion of myocardium was necessary to successfully defibrillate.

Optogenetic defibrillation after acute myocardial infarction. To experimentally assess the feasibility of optogenetic defibrillation in a scenario clinically more relevant to the underlying causes of life-threatening arrhythmia in humans, we determined whether infarct-related arrhythmia could be terminated by light. Therefore, hearts from ChR2 mice were perfused ex vivo with standard Tyrode's solution, and the left anterior descending coronary artery was ligated (Figure 2A, arrow). This led to acute myocardial ischemia, which was proven by ST-segment elevation in the ECG (Figure 2B) and by the lack of local perfusion documented by dye injection after the experiment (Figure 2A). Prolonged ventricular arrhythmia could be induced by electrical pacing in 6 of the 12 hearts investigated. In these 6 hearts we found that a single light pulse ( 1 second, $0.4 \mathrm{~mW} / \mathrm{mm}^{2}, 143 \mathrm{~mm}^{2}$ ) could terminate arrhythmia (Figure 2B) with a success rate of $88 \% \pm 0.04 \%$ in contrast to the significantly lower rate of spontaneous termination in the absence of illumination ( $27 \% \pm 0.07 \%, P=0.03$; Figure $2 \mathrm{C})$.

Optogenetic defibrillation in WT hearts after gene transfer. After establishing the feasibility of optogenetic defibrillation in transgenic mice, we sought to test the same approach in nontransgenic hearts after gene transfer in vivo, since this would be required for therapeutic applications in humans. We previously used adenoassociated virus (AAV) with tropism toward cardiomyocytes systemically injected into WT mice and found ChR2 expression in approximately $58.2 \%$ of cardiomyocytes 4 to 8 weeks later, enabling optogenetic cardiac pacing (6). With the same approach, 1 year after AAV injection we found uniform ChR2 expression throughout the ventricles (Figure 3A) and in all myocardial layers (Figure 3B), localized to the sarcolemma and the T-tubule system of cardiomyocytes (Figure $3 \mathrm{C}$ ). In vivo optogenetic pacing experiments $(5,6)$ in these hearts demonstrated stable ventricular pacing 1 year after the AAV injection with a threshold of $0.64 \pm 0.20$ $\mathrm{mW} / \mathrm{mm}^{2}$ (Figure 3D; $10 \mathrm{~ms}$ pulse duration, $15 \mathrm{~mm}^{2}$ illuminated area, $n=3$ ). This value is similar to our previously reported data for optogenetic pacing 4-8 weeks after injection of AAV (6). Importantly, we also tested optogenetic defibrillation ex vivo 1 year after the AAV injection. Therefore we induced ventricular arrhythmia after low $\mathrm{K}^{+}(2 \mathrm{mM})$ and pinacidil perfusion and found that illumination ( 1 second, $0.4 \mathrm{~mW} / \mathrm{mm}^{2}, 143 \mathrm{~mm}^{2}$ ) of the anteroseptal epicardium reliably terminated ventricular arrhythmia in all 3 tested mice (Figure 3E).

Optogenetic defibrillation via epicardial illumination in a model of a patient heart. In order to explore whether our findings in mice could be translated to human hearts for potential clinical applications, we conducted computer simulations of optical defibrillation by epicardial illumination in the heart of a patient suffering recurrent VT. The model (Supplemental Figure 2) was reconstructed from a late gadolinium enhancement MRI scan of a patient with myocardial infarction and VT episodes that required defibrillator implantation. Consistent with clinical observations, rapid electrical pacing from the ventricular apex in this model induced infarct-related, sustained VT (cycle length: 350 ms; Supplemental Figure 3 and Supplemental Video 1), which was evident in the pseudo-ECG (Figure 4A). Based on expression levels in our previous reports on AAV gene transfer (6), we simulated expression of ChR2 in 58.2\% of the cardiomyocytes (Supplemental Figure 4) using an optogenetic modeling framework (13) validated by comparison with in vitro recordings from ChR2-expressing cardiomyocyte syncytia (14). The ChR2 

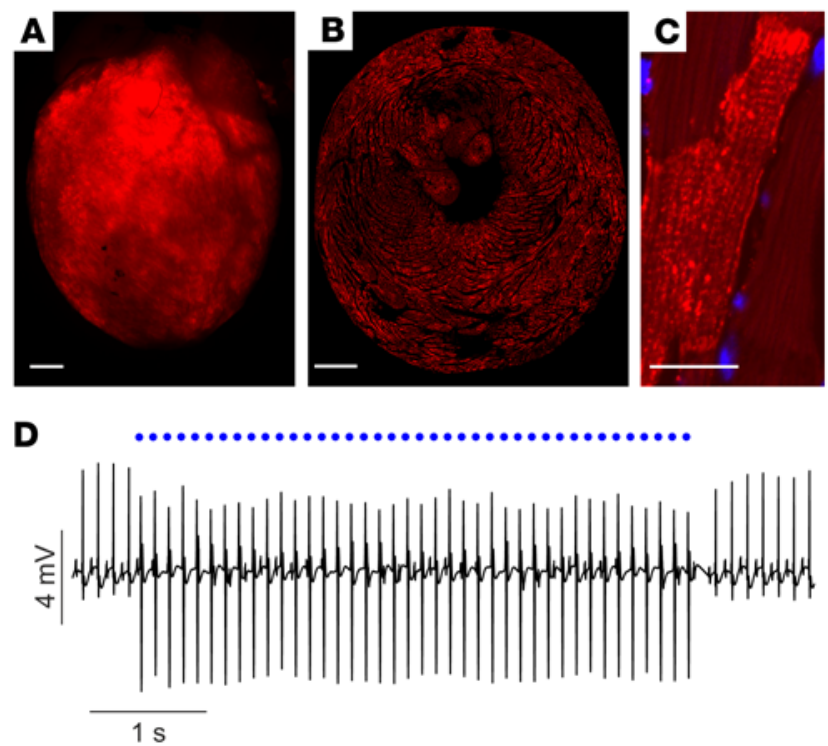

$\mathbf{E}$

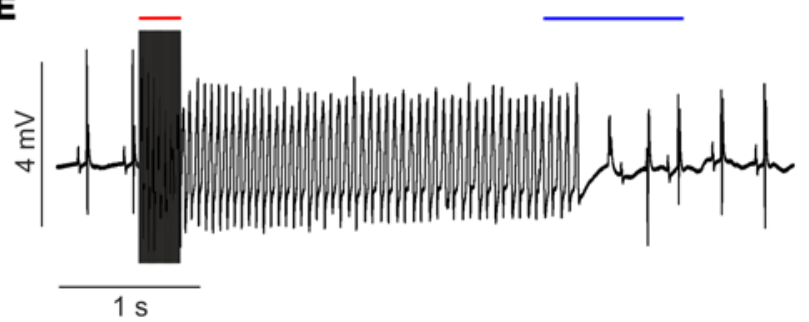

photocurrent model (15) was calibrated to match measurements from AAV-transfected mouse cardiomyocytes (6).

We have recently shown in computer stimulations that for optogenetic defibrillation of a structurally normal heart by simultaneous epicardial and endocardial illumination, red light and increased opsin light sensitivity (increased 2.2-fold vs. normal ChR2) are needed (16). These changes are consistent with the biophysical properties of red-shifted ChR2 variants $(17,18)$ and the CatCh variant of ChR2 (19), respectively. Accordingly, to assess the feasibility of light-based defibrillation in the more complex setting of a patient heart with structural and electrophysiological heterogeneities, we performed simulations with the same optogenetic parameters. However, epicardial-only illumination was simulated, because the feasibility of endocardial illumination is questionable. Our simulations revealed that exclusive illumination from the epicardium with red light (1 second, $10 \mathrm{~mW} / \mathrm{mm}^{2}$, $669 \mathrm{~nm}$ ) effectively terminated the sustained VT (Figure 4, B and C, and Supplemental Video 2).

Critical biophysical determinants for optogenetic defibrillation. Next, we investigated the critical factors underlying successful optogenetic defibrillation by analyzing membrane potential $\left(V_{m}\right)$ traces from epicardial, midmyocardial, and endocardial sites during simulated illumination (Figure 5A). Our analysis revealed simultaneous transmural excitation upon illumination, followed by strong diastolic depolarization $(\sim-40 \mathrm{mV})$ that affected all layers (Figure $5 \mathrm{~B}$ ) and completely abrogated $\mathrm{Na}^{+}$channel excitability, even in the endocardial layer (Figure 5C). Therefore, action potential initiation during illumination became purely $\mathrm{Ca}^{2+}$ current-dependent (Figure $5 \mathrm{C}$ ), resulting in a critical decrease in
Figure 3. Optogenetic pacing and defibrillation in hearts 1 year after in vivo gene transfer. (A) Epicardial mCherry fluorescence signal of a mouse heart 16 months after systemic injection of ChR2-mCherry AAV. (B) Section through the left ventricle showing transmural mCherry fluorescence. (C) Membrane-bound ChR2-mCherry signals in a ventricular cardiomyocyte. (D) Pulsed illumination (blue dots, $470 \mathrm{~nm}, 10 \mathrm{~ms}, 0.36 \mathrm{~mW} / \mathrm{mm}^{2}, 15$ $\mathrm{mm}^{2}, n=3$ ) of the left ventricle in vivo induced ectopic ventricular pacing, as shown in the ECC (black). (E) VT episode was terminated by epicardial illumination ( $470 \mathrm{~nm}, 1$ second, $0.4 \mathrm{~mW} / \mathrm{mm}^{2}, 143 \mathrm{~mm}^{2}, n=3$ ) of the ventricles. Scale bars: $1 \mathrm{~mm}$ (A and B) and $20 \mu \mathrm{m}$ (C); nuclear staining in blue.

the excitability of the illuminated myocardial wall. Importantly, the deepest layer in which red light depolarized at least $75 \%$ of all cardiomyocytes beyond the threshold to inactivate $90 \%$ of $\mathrm{Na}^{+}$ channels $\left(V_{m}=-65.8 \mathrm{mV}\right)$ was $6 \mathrm{~mm}$ from the epicardium (Figure $5 \mathrm{D}$, red arrow). Consequently, the wavefront emerging from the isthmus of the re-entrant circuit, located in the nonilluminated ventricular septum, could not propagate through the optogenetically depolarized right ventricular free wall, thereby leading to VT termination (Figure 5E and Supplemental Video 3).

To verify that transmural depolarization is critical for optogenetic defibrillation, we simulated epicardial illumination with blue light, which is much more attenuated by heart tissue (Supplemental Figure 5) (20). In fact, blue light (488 nm) triggered an initial epicardial excitation, which propagated transmurally and induced the above-defined degree of depolarization to block $\mathrm{Na}^{+}$channels only within $1 \mathrm{~mm}$ of the epicardial surface (Figure 5D, blue arrow, and Supplemental Figure 6A). Therefore, the excitation wavefront emerging from the septum was able to propagate through the endocardial layer of the right ventricular free wall, sustaining re-entrant propagation (Supplemental Figure 6, B and C, and Supplemental Video 2 and 3).

Modeling of pericardial illumination. Since illumination of the pericardium, rather than direct application of light to the epicardium, will be practically more feasible, we performed additional calculations to assess how the tissue layers surrounding the human heart would attenuate light. First, we considered layers of epicardial fat and pericardium assuming uniform thickness based on values previously reported for these layers in humans (4.1 and $1.7 \mathrm{~mm}$, respectively) $(21,22)$. We found that the intensity of a pericardial light stimulus would need to be 4.7 -fold higher than the target intensity on the epicardium in order to overcome attenuation in the fibrous and adipose layers covering the heart (Supplemental Figure 7A). Since individual and regional variability in epicardial fat thickness has been reported (21), we analyzed the consequences and found a required increase in pericardial light stimulus strength from approximately 2 -fold in the absence of fat to approximately 10 -fold when the adipose tissue layer was $8 \mathrm{~mm}$ thick (Supplemental Figure 7B).

\section{Discussion}

Herein we demonstrate effective optogenetic defibrillation in the intact mouse heart by epicardial illumination. To enable these experiments, we had to establish a new approach to reliably induce sustained ventricular arrhythmia in the small mouse heart, which normally lasts only a few seconds in vivo (12). Our approach is to perfuse explanted mouse hearts with low- $\mathrm{K}^{+}$solution, which is known to promote arrhythmias (23), and pinacidil, which activates 
A

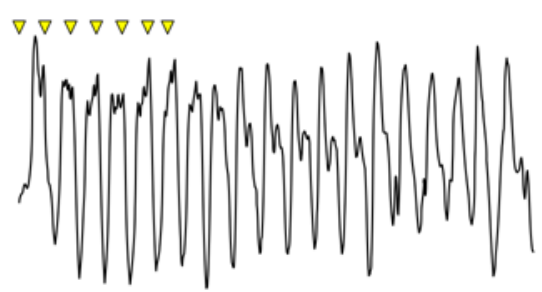

B

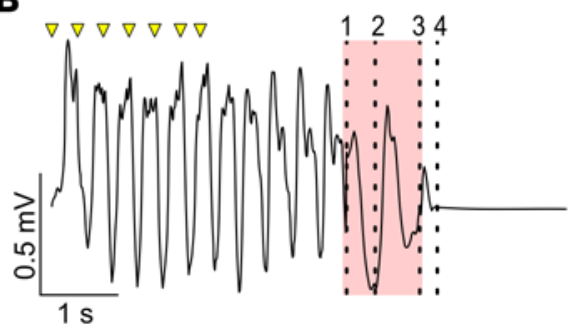

C

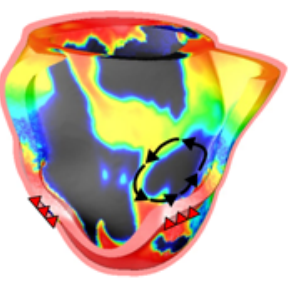

(1) $t=4.01 \mathrm{~s}$

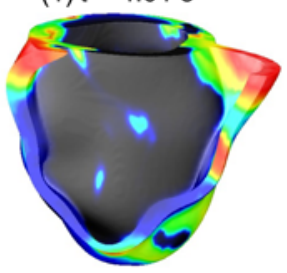

(3) $5.01 \mathrm{~s}$

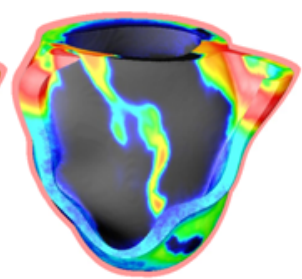

(2) $4.40 \mathrm{~s}$

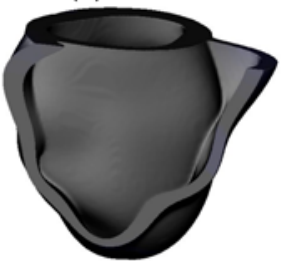

(4) $5.25 \mathrm{~s}$
Figure 4. Optogenetic defibrillation in a model of a patient heart with infarct-related VT. (A and B) Pseudo-ECG signals for model configurations with no illumination (A) and epicardial illumination with red light (669 nm, $10 \mathrm{~mW} / \mathrm{mm}^{2}, 1$ second, red box, B). VT was induced by electrical stimulation (yellow triangles). (C) Snapshots of $V_{m}$ distribution during the response to red light. Timings (from start of simulation) correspond to dashed lines in $\mathbf{B}$. The right ventricular free wall is cut away to facilitate visualization. Black arrows indicate propagation of reentry during VT (see also Supplemental Figure 3 and Supplemental Videos 1 and 2); red triangles indicate initial excitations induced directly by optogenetic stimulation. the ventricular Kir6.2/SUR2A $\mathrm{K}_{\mathrm{ATP}}$ channels (24) and thus mimics pro-arrhythmic effects of ischemia, including action potential shortening and dispersion of repolarization and refractoriness (25). The ex vivo retrograde perfusion of the coronary arteries in the Langendorff configuration has the distinct advantage of constant oxygen and nutrition supply even during long episodes of sustained ventricular arrhythmia, enabling multiple defibrillation attempts for systematic analysis of defibrillation success rate.

Epicardial illumination proved to be highly efficient in all ChR2-expressing mouse hearts tested in terminating ventricular arrhythmias, the ECG patterns of which resembled those of VT, torsades de pointes, and VF. By comparing light-induced and spontaneous arrhythmia termination in ChR2-expressing hearts and by investigating control hearts without ChR2, we proved unequivocally that neither illumination nor ChR2 expression per se is sufficient for arrhythmia termination. Instead, optogenetic defibrillation is achieved by light-induced depolarization of ChR2-expressing cardiomyocytes. We found that optogenetic defibrillation required higher light intensities, longer durations, and larger illumination sizes compared with optogenetic pacing (5), reflecting the clear mechanistic difference between pacing, for which local depolarization is sufficient, and defibrillation, which requires depolarization of a much larger tissue volume (9). This requirement of defibrillation was proven in earlier work by selective coronary injection of potassium chloride, demonstrating that $\mathrm{VF}$ in canines could only be terminated when a critical amount of myocardium was depolarized (9). Our findings are consistent with these experiments because diminishing the illumination area reduced the efficacy of optogenetic defibrillation.

Although our experimental work here demonstrated the efficacy of optogenetic defibrillation after acute myocardial infarction and after AAV-based ChR2 gene transfer of WT mice, it is difficult to extrapolate these results to the human heart. Thus, the next step will be the extension of this technology to preclinical large-animal models with electrical and structural defects comparable to the human heart. As a preliminary proof-of-concept exploration in humans, we simulated optogenetic defibrillation in a human model of infarct-related VT (26). Our group has recently demon- strated the in silico approach to simulate this type of arrhythmia and its predictive power and clinical relevance, by predicting, with high accuracy and reliability, postinfarction risk of sudden cardiac death in a cohort of 41 patients (27). Here, in contrast to our recent modeling of optogenetic defibrillation in structurally normal ventricles (16), we exclusively illuminated the epicardial surface. This is because intracavitary optogenetic stimulation of the endocardium would be extremely challenging because of the presence of light-absorbing blood, the need for anticoagulation, and the difficulty of implanting light-emitting diodes (LEDs) within the densely trabeculated endocardium of the beating heart without compromising ventricular function or hemodynamic stability. Conversely, illumination of the epicardium in the human heart could be achieved by leveraging new developments in optoelectronics, such as light sources integrated in cell-scale injectable devices (28) or stretchable integumentary membranes, which have been proven useful for illumination of the heart (29). Moreover, because direct epicardial implantation of light sources will be associated with risks of infection, pericarditis, friction, or coronary artery damage, we anticipate that illumination in the human heart would be more feasible from the pericardial surface. Thus, we performed mathematical analysis to approximate the extent of light attenuation in the pericardium and epicardial fat and found that the intensity of light applied to the pericardium would need to be increased approximately 5 -fold compared with direct epicardial illumination. However, excessive epicardial fat would greatly attenuate light and thus diminish the effectiveness of optogenetic defibrillation by pericardial illumination.

The new mechanistic insights presented here suggest that ventricular arrhythmia can be terminated not only by electrical shock-induced brief excitation, but also by a steady-state optogenetic modulation of electrical activity involving transmural depolarization and $\mathrm{Na}^{+}$channel inactivation. Importantly, our simulations clearly demonstrate that transmural depolarization depends critically on effective light penetration. This parameter is influenced greatly by the wavelength dependency of light penetration and, to a lesser degree, by ChR2 light sensitivity, photocurrent, or expression levels. Thus sufficient transmural depo- 
A
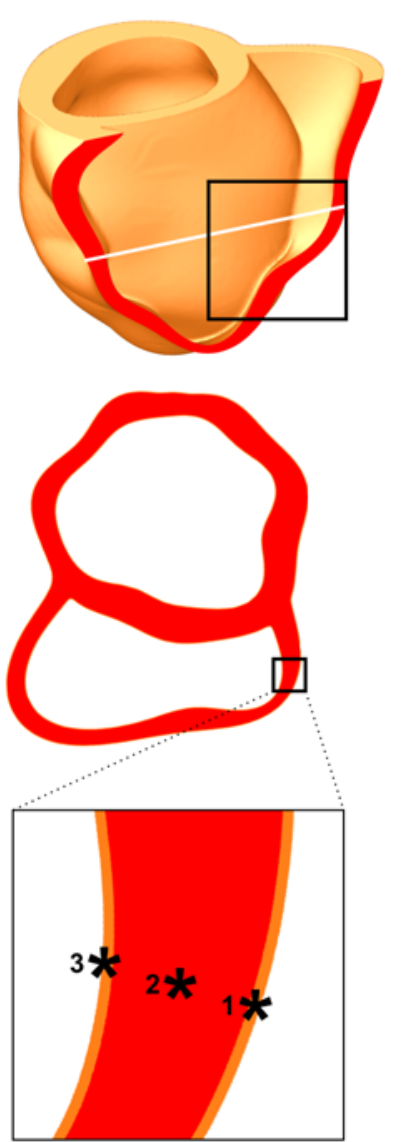

B

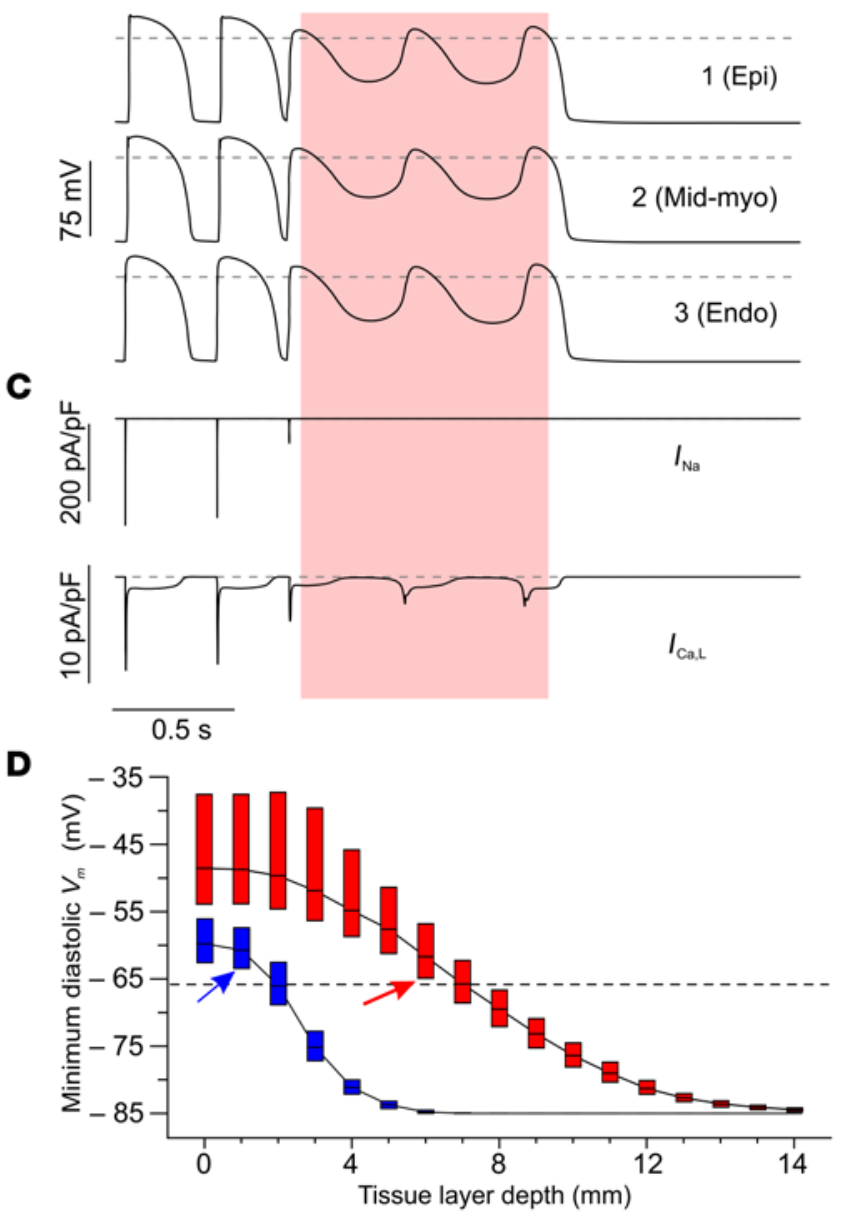

E

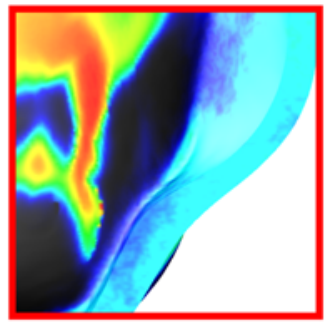

$t=4.32 \mathrm{~s}$

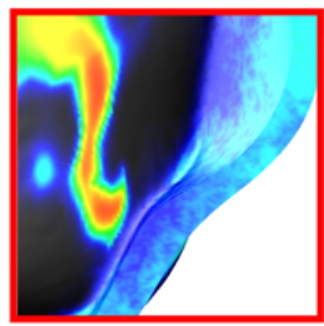

$t=4.37 \mathrm{~s}$

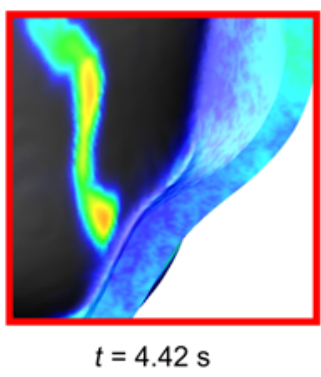

$-85 V_{m}(\mathrm{mV})+35$

Figure 5. Mechanism of optogenetic defibrillation in the human heart. (A) Illustration of the locations of sites 1-3 on the right ventricular posteroseptal wall. (B) $V_{m}$ traces from sites 1-3 during epicardial illumination with red light ( $669 \mathrm{~nm}, 1$ second, $10 \mathrm{~mW} / \mathrm{mm}^{2}$, red box) Dashed lines indicate $V_{m}=0$. (C) Ionic $\mathrm{Na}^{+}\left(I_{\mathrm{Na}}\right)$ and L-type $\mathrm{Ca}^{2+}\left(I_{\mathrm{Ca}, \mathrm{L}}\right)$ currents underlying $V_{m}$ from endocardial site 3 in B. (D) Minimum diastolic $V_{m}$ values during illumination with blue (488 $\mathrm{nm}$ ) and red light as a function of depth in the ventricular free walls. Upper quartile, median, and lower quartile values are shown. Blue and red arrows indicate the deepest tissue layers in which more than $75 \%$ of cardiomyocytes had a minimum diastolic $V_{m}$ exceeding the threshold for $90 \%$ inactivation of $\mathrm{Na}^{+}$current $\left(V_{m}=-65.8 \mathrm{mV}\right.$, dashed line). (E) Zoomed-in snapshots of $V_{m}$ distribution during the illumination at the indicated time points from start of simulation (see also Supplemental Video 3). Snapshots were taken from the region shown in $\mathbf{A}$ (black rectangle, in top panel).

larization at low light intensities will be facilitated by exploiting recent advances in optogenetics, like red-shifted and hypersensitive ChR2 variants (17-19).

The fact that optogenetic defibrillation in human diseased hearts is feasible using only epicardial illumination and that cardiac gene expression via AAV in humans is safe (30) suggests the tantalizing possibility that clinical applications might be feasible. Importantly, we were able to demonstrate effective pacing and defibrillation of mouse hearts by epicardial illumination even more than 1 year after the gene transfer. The great advantage of optogenetic defibrillation is that this would be, in contrast to electrical shocks, pain-free, because ChR2 expression would be restricted to cardiomyocytes by cardiomyocyte-specific AAV vector capsids (30) or promoters (31). Notably, AAV-mediated transduction will result in spatial heterogeneity of ChR2 expression, which is not expected to be pro-arrhythmic per se since ChR2 has neither a leak current in the absence of illumination nor does it affect the electrophysiological properties of cardiomyocytes (5). During illu- mination, however, non-uniformities in either ChR2 expression or light application could result in heterogeneous excitation patterns, which might have secondary pro-arrhythmic consequences (e.g., reinitiation of $\mathrm{VT} / \mathrm{VF}$ ) in addition to the primary effect of optogenetic defibrillation. Fortunately, in contrast to electrical shocks, light stimuli used for optogenetic defibrillation have a much higher degree of flexibility, because spatially and temporally shaped light patterns minimizing secondary pro-arrhythmic effects could be used. Moreover, unlike defibrillation shocks, sequential light pulses can be applied repetitively, as these do not require the charging of a capacitator to generate high-voltage fields. In fact, our experimental results in mouse hearts showed that 4 consecutive light pulses instead of 1 increased the termination rate from $85 \%$ to $97 \%$. Importantly, the optogenetic modeling approach presented here makes it possible to predict in silico the effectiveness of novel red-shifted ChR2 variants, gene transfer strategies, or illumination technologies before conducting large animal experiments in vivo and before proposing individualized clinical trials, 
e.g., for therapy-resistant patients who experience frequent painful electrical shocks from implanted defibrillators.

\section{Methods}

Animals. Experiments on transgenic ChR2-expressing mice were performed with 23 males (aged 4-15 months, median 6 months) and 27 females (aged 3.5-14.5 months, median 4.5 months) from a previously established mouse line (5) expressing ChR2 with the H134R mutation (32) under the control of the chicken $\beta$-actin (CAG) promoter and backcrossed at least 10 generations onto a CD1 genetic background. The CAG-EGFP mouse line was reported before (5), backcrossed at least 10 generations onto a CD1 genetic background, and we used five 2.5- to 3.5-month-old females. From the CD1 WT strain (Charles River) we used 7 female mice 6.5 to 12 months old as controls and three 10-weekold female mice for ChR2 gene transfer by systemic injection of AAV.

Optogenetic defibrillation. Mice were heparinized and sacrificed by cervical dislocation. Hearts were explanted and perfused in Langendorff configuration with Tyrode's solution containing (in mM) 140 $\mathrm{NaCl}, 5.4 \mathrm{KCl}, 1.8 \mathrm{CaCl}_{2}, 2 \mathrm{MgCl}_{2}, 10$ glucose, and 10 HEPES ( $\mathrm{pH} 7.4$, adjusted with $\mathrm{NaOH}$ ). A bipolar cardiac electrogram was recorded with a silver chloride electrode placed at the right atrium and a metal spoon under the apex of the heart using a bio-amplifier recording system (PowerLab 8/30, Animal Bio Amp ML 136, LabChart 7.1 software, AD Instruments). Electrical stimulation (1-10 mA, $2 \mathrm{~ms}$ ) was applied via a bipolar silver chloride electrode $(<3 \mathrm{~mm}$ distance) placed epicardially. The local effective refractory period (ERP) was determined using an S1-S2 protocol, with 6 stimuli at a cycle length of 150 $\mathrm{ms}$ (S1) followed by a premature stimulus (S2) with a variable delay $(<150 \mathrm{~ms})$ that was decreased in $5-\mathrm{ms}$ increments. The shortest delay for successful S2 pacing was defined as the local ERP. The electrode was relocated until an epicardial site with a low local ERP $(<55 \mathrm{~ms})$ was identified, because we observed that pacing from such locations was the most reliable way to induce ventricular arrhythmia in mouse hearts. Application of either electrical burst stimulation $(50 \mathrm{~Hz}, 20-50$ pulses) or an S1-S2 protocol with an S2 delay exceeding the local ERP by less than 5 ms evoked free-running ventricular arrhythmia. In standard Tyrode's solution, arrhythmia episodes often terminated spontaneously after less than 15 seconds. Because sustained arrhythmias are required to test optogenetic defibrillation, we lowered the potassium concentration to $2 \mathrm{mM}$ and added $100 \mu \mathrm{M}$ pinacidil, which enabled induction of sustained ( $>30$ seconds) ventricular arrhythmia. Notably, optogenetic defibrillation was attempted in all cases at least 3 seconds after induction, which led to inclusion of sustained arrhythmia only and exclusion of self-terminating VTs, most of which were shorter than 3 seconds. Illumination of the anterior ventricular epicardium (Supplemental Figure 1A) was performed with a macroscope (MVX10, Olympus) equipped with a $1 \times$ objective (MVPLAPO1x, numerical aperture 0.25) using 470-nm LEDs (LEDC5 and LEDD1, Thorlabs, or GCS-0470-50-A0510 and BLS-13000-1, Mightex) attached to the epifluorescence port. LED pulse duration and intensity were controlled and sampled with the bio-amplifier recording system. The spatial extent of the illuminated area was varied using the zoom function of the macroscope (Supplemental Figure 1A). Light intensity was calibrated by measuring the light power with a power meter (PM100 and S130A, Thorlabs) and dividing by the illuminated area. The maximum light intensities tested were $0.4,1.5$, and $1.5 \mathrm{~mW} / \mathrm{mm}^{2}$ for $143-, 29-$, and $15-\mathrm{mm}^{2}$ large circular areas, respectively.
We assessed defibrillation success rates by conducting multiple defibrillation attempts per heart using 2 different protocols as follows. (a) Single-light-pulse protocol (Supplemental Figure 1B): VT/VF episodes were allowed to stabilize for 3,5 , or 10 seconds after induction. Subsequently, 1 single light pulse ( 1 second, $0.4 \mathrm{~mW} / \mathrm{mm}^{2}, 143 \mathrm{~mm}^{2}$ ) was applied, and we determined whether it terminated the arrhythmia within 4 seconds after the start of illumination (green box in Supplemental Figure 1B); otherwise, rescue defibrillation was performed. Spontaneous termination rate was analyzed during the same time period but without illumination (Figure 1B, black). (b) Four-light-pulse protocol (Supplemental Figure 1C): VT/VF episodes were allowed to stabilize for 3-15 seconds after induction. Subsequently, 4 light pulses with identical illumination parameters were applied with a 1- to 5-second delay in between, and we determined whether the arrhythmias were terminated between the onset of the first light pulse and 3-10 seconds after the last illumination (green box in Supplemental Figure 1C); otherwise rescue defibrillation was performed. For control experiments, the longest illumination protocols were applied to hearts from transgenic mice expressing EGFP under the control of the chicken $\beta$-actin promoter (5) and from CD1 WT mice (Figure 1C, green, red). The rate of spontaneous termination in ChR2 mice was assessed during the maximum duration of the 4-light-pulse protocol (Figure 1C, black) but without illumination. The efficacy of the different illumination parameters (pulse duration, illuminated area, and intensity; Figure 1, D and E) was analyzed using the 4-light-pulse protocol with identical illumination parameters for each of the 4 pulses.

The rescue defibrillation procedure included sequential or simultaneous application of high-intensity illumination $\left(145 \mathrm{~mm}^{2}\right.$, 0.4 to $0.9 \mathrm{~mW} / \mathrm{mm}^{2},>3$ seconds), mechanical force applied to the ventricles by tweezers, and/or electrical antitachycardia pacing (50 $\mathrm{Hz},>20$ pulses). If this procedure did not terminate the arrhythmia, the heart was temporarily perfused with normal Tyrode's solution and, if this restored normal rhythm, optogenetic defibrillation experiments were reattempted.

Acute myocardial infarction. The left anterior descending coronary artery of explanted hearts was ligated with a Prolene 8-0 thread (Ethicon). Ventricular arrhythmias were induced as reported above, but during perfusion with standard Tyrode's solution. This induced prolonged VT/VF episodes ( $>10$ seconds) in 6 of 12 hearts. In these hearts, optogenetic defibrillation was tested 5 seconds after induction using the single-light-pulse protocol (Supplemental Figure 1B), as described above. To verify complete artery ligation, hearts were coronary-perfused after the experiment with the food dye Patent Blue V E131 (Brauns-Heitmann), and macroscopic images were taken (see below).

$A A V$-based gene transfer and optical cardiac pacing. Systemic injection of AAV was performed as reported earlier (6). Briefly, the AAV9-CAG-hChR2(H134R)-mCherry virus consisting of AAV9 virus capsid and AAV2 virus DNA was obtained from the Penn Vector Core (University of Pennsylvania) and injected into the jugular vein of WT mice anesthetized by inhalation of $1.5 \%$ isoflurane and $\mathrm{O}_{2} / \mathrm{N}_{2} \mathrm{O}$ $(50 \% / 50 \%)$. For this purpose, a 5 -mm-long cervical incision was performed, $2 \times 10^{11} \mathrm{AAV}$ particles diluted in $100 \mu \mathrm{l}$ of PBS were slowly ( 10 seconds) injected, bleeding was stopped with forceps and surgical

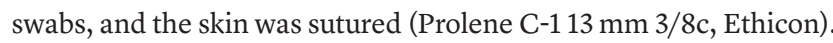

For optical cardiac pacing in vivo, mice were intubated and ventilated with $1.5 \%$ to $2.5 \%$ isoflurane and $\mathrm{O}_{2} / \mathrm{N}_{2} \mathrm{O}(50 \% / 50 \%) 16$ months 
after the virus injection. To expose the beating heart, the rib cage was carefully removed as reported earlier $(5,6)$. Cardiac ECGs were recorded from a needle electrode placed at the right front leg and a spoon placed below the heart for stabilization using the bio-amplifier recording system (see above). Heart rates were adjusted to 350 to 450 bpm by varying isoflurane concentration. Optical pacing of the epicardial surface of the left ventricle was performed at a constant frequency 30 to 50 bpm above basal heart rate using the macroscope and LED system (see above). At each light intensity, a train of 40 light pulses was applied, and the threshold for optical pacing was defined as the minimal light intensity required to achieve 1:1 stimulation for the last 20 pulses. After in vivo optical pacing, hearts were explanted during anesthesia and perfused in Langendorff configuration. Optogenetic defibrillation was then tested as described above.

Histology and immunofluorescence. High-resolution macroscopic images were recorded from explanted and perfused hearts with an AxioZoom.V16 macroscope equipped with a PlanApoZ $\times 1.0$ objective lens, a 43 HE filter set, and an AxioCam MRm camera with ZEN 2012 software (all Zeiss). Images of the curved epicardium were generated using Z-stacks and the extended focus module (Zeiss). Hearts were fixated by perfusion with $4 \%$ paraformaldehyde, dehydrated in $20 \%$ sucrose, cryopreserved, and sectioned in 10- $\mu \mathrm{m}$-thick slices with a cryotome (Cryostat CM3050 S, Leica). Heart slices were permeabilized with $0.2 \%$ Triton X (Sigma-Aldrich) for 20 minutes. Nuclear staining was performed with $0.1 \%$ Hoechst 33342 (Sigma-Aldrich) for 1 hour at room temperature. Microscopic pictures were taken with inverted fluorescence microscopes (Axiovert 200M) equipped with the Apotome section module, a $\times 20$ or $\times 40$ Plan-Apochromat objective, AxioCam MRc or MRm camera, ZEN 2012 or AxioVision software, a 64 HE filter set (all Zeiss), and an mCherry filter set (F46008, AHF Analysentechnik).

Geometrical model of the diseased human ventricles. We used a computational model of the diseased human ventricles reconstructed from clinical late gadolinium-enhanced MRI (LGE-MRI) scans of a patient with post-myocardial infarction VT (33). The modeling approach has been previously validated in infarct-related VT inducibility studies $(12,34,35)$. Our laboratory pipeline for cardiac model reconstruction has been described in detail in previous work (12, 34-37). Briefly, anatomical landmark points on the epicardial and endocardial walls were identified manually in each LGE-MRI slice. These points were used as input data for previously described interpolation software, which was used to recreate a $3 \mathrm{D}$ rendering of the cardiac surfaces. To represent the patient's infarct geometry, tissue within the ventricular contours on each 2-dimensional LGE-MRI slice was classified as diseased or healthy via signal thresholding. Diseased tissue was then classified as either peri-infarct zone (PIZ) or infarct using a validated full-width half-maximum approach (38). 3D boundaries between the 3 tissue regions were reconstructed using a shape-based interpolation approach (39) that has been validated for use in characterizing myocardial scar (40). We then combined infarct and PIZ contours with the rendering of ventricular geometry and used a fully automatic octreebased technique (41) to generate a boundary-fitted, locally refined, smoothed, and conformal 3D mesh (Supplemental Figure 2) suitable for finite element modeling of cardiac electrophysiology. The ventricular mesh had 2,110,030 points and 2,687,615 volumetric elements. Finally, myocardial fiber orientations were assigned throughout this mesh using a previously validated rules-based approach (42).
Simulation of cardiac electrophysiology and numerical methods. Electrophysiology of normal and diseased human cardiac tissue was represented using an extensively validated approach (35). Briefly, cell- and tissue-scale properties were assigned to all 3 regions in the ventricular mesh: normal myocardium, PIZ, and infarct. At the cell scale, the infarcted region was modeled as electrically passive. In the PIZ and normal myocardium, action potential dynamics were represented via a validated human-specific model (43). In the PIZ, specific modifications to this model were incorporated to represent electrophysiological remodeling observed in patch-clamped myocytes harvested from the infarct border zone: fast sodium current $\left(I_{\mathrm{Na}}\right)$ was decreased by $62 \%$ (44), L-type calcium current $\left(I_{\mathrm{Ca}, \mathrm{L}}\right)$ was decreased by $68 \%$ (45), and delayed rectifier potassium currents $\left(I_{\mathrm{Kr}}\right.$ and $\left.I_{\mathrm{Ks}}\right)$ were decreased by $80 \%$ and $70 \%$, respectively (46). Consequently, the PIZ action potential differed from that in the normal myocardium: $+16.1 \%$ duration, $-42.2 \%$ upstroke velocity, and $-42.8 \%$ amplitude. These changes were consistent with previously reported values $(47,48)$. At the tissue scale, the infarcted region was modeled as nonconductive. In normal myocardium, longitudinal and transverse intracellular conductivity values were scaled to 0.255 and $0.0775 \mathrm{~S} / \mathrm{m}$, respectively. This resulted in longitudinal and transverse conduction velocities $\left(\mathrm{CV}_{\mathrm{L}}=59.9\right.$ and $\mathrm{CV}_{\mathrm{T}}$ $=33.5 \mathrm{~cm} / \mathrm{s}$, respectively) consistent with values measured in experiments with human ventricular tissue $(49,50)$. In the PIZ, transverse intracellular conductivity was reduced by $90 \%$ to represent connexin-43 remodeling observed in the infarct border zone (51). Because of this change and differences in cell-level electrophysiology (as described above), PIZ conduction velocities were lower than in normal myocar$\operatorname{dium}\left(\mathrm{CV}_{\mathrm{L}}=49.2 \mathrm{~cm} / \mathrm{s}, \mathrm{CV}_{\mathrm{T}}=4.16 \mathrm{~cm} / \mathrm{s}\right)$ and had a larger anisotropy ratio (11.8:1 vs. 1.79:1). Excitation propagation in the ventricular model was modeled via the monodomain formulation as implemented in the CARP software (Johns Hopkins University, Université de Bordeaux, Medizinische Universität Graz) $(52,53)$. As described previously (52), a leapfrogging approach was used to couple the monodomain partial differential equation with the system of ordinary differential equations that modeled cell-level phenomena. Our previous studies have described rigorous validation of this computational framework against experimental measurements $(14,34,54-56)$. All simulations were executed on 24 Intel Haswell CPUs $(2.5 \mathrm{GHz})$ on the parallel computing resource at the Maryland Advanced Research Computing Center. The total wall time required to simulate 1 second of electrophysiological activity in the ventricular model was about 1 hour 40 minutes.

Simulation of channelrhodopsin expression and illumination. To simulate optogenetic transduction of the human ventricles via viral gene delivery, we used our previously validated computational modeling framework $(13,14,57)$. Channelrhodopsin expression was modeled by incorporation of a photocycle model (15) in 58.2\% of myocardial nodes (normal + PIZ) with a diffuse spatial pattern (Supplemental Figure 4). This value corresponded to the reported average percentage of ChR2-expressing cardiomyocytes in mouse hearts after systemic AAV injection (6). The ChR2 channel conductance parameter was calibrated to $0.17 \mathrm{mS} / \mathrm{cm}^{2}$, resulting in a peak photocurrent of $5.04 \mathrm{pA} /$ $\mathrm{pF}$ when a simulated myocyte clamped to $V_{m}=-52 \mathrm{mV}$ was subjected to blue illumination $\left(5 \mathrm{~mW} / \mathrm{mm}^{2}\right)$. This value matched ( $<1 \%$ error) recorded peak photocurrent in patch-clamped ChR2-positive myocytes from our earlier study (6). ChR2 light sensitivity was adjusted to the CatCh variant $(17,19)$ by increasing the model rate parameters controlling the dark- and light-adapted transitions between closed and 
open states (i.e., $k 1$ and $k 2$ in the Williams et al. model; ref. 15) by 2.2 fold. This resulted in a half-maximal optogenetic activation level (i.e., threshold irradiance to elicit $50 \%$ of maximal $I_{\mathrm{ChR} 2}$ ) of $0.4 \mathrm{~mW} / \mathrm{mm}^{2}$, which was consistent with the reported value for CatCh (58). The expression for ChR2 photocurrent was:

$$
I_{\mathrm{ChR} 2}=g_{\mathrm{ChR} 2}\left(O_{1}+\gamma O_{2}\right)\left(V_{m}-E_{\mathrm{ChR} 2}\right)
$$

(Equation 1)

where $g_{\text {ChR2 }}$ was the maximal channel conductance $\left(0.17 \mathrm{mS} / \mathrm{cm}^{2}\right)$, $\gamma=0.1$ was the conductance ratio between dark- and light-adapted open channel states $\left(O_{1}\right.$ and $O_{2}$, respectively), and $E_{\text {ChR2 }}=0 \mathrm{mV}$ was the ChR2 reversal potential. The ordinary differential equations controlling light- and voltage-dependent rates of change in $O_{1}$, $\mathrm{O}_{2}$, and other model state variables can be found in previous publications $(13,15,57)$. At the organ scale, we used a finite element approximation of the steady-state photon diffusion equation to model light attenuation due to scattering and energy absorption in the ventricular myocardium $(13,57)$. The corresponding partial differential equation, which assumes isotropic absorption and homogenous scattering, is given by:

$D \nabla^{2} E_{e}(r)-\mu_{\mathrm{a}} E_{e}(r)=-w(r)$

(Equation 2)

where $E_{e}$ and $w$ are, respectively, the distributions of irradiance and photon sources at each point $r ; \nabla^{2}$ is the Laplace operator; $D$ is the diffusivity of light in cardiac tissue; and $\mu_{\mathrm{a}}$ is the light absorption rate. As described previously (57), $w$ is set to zero and the equation is solved with Dirichlet boundary conditions derived from the placement of simulated light sources. We validated this approach in earlier work concerned with simulation of optical mapping experiments (20). Optical parameters of myocardium were derived from experimentally measured values for cardiac tissue (59) and depended on whether the applied illumination was blue $\left(\lambda=488 \mathrm{~nm}, D=0.18 \mathrm{~mm}, \mu_{\mathrm{a}}=0.52\right.$ $\left.\mathrm{mm}^{-1}\right)$ or red $\left(\lambda=669 \mathrm{~nm}, D=0.34 \mathrm{~mm}, \mu_{\mathrm{a}}=0.10 \mathrm{~mm}^{-1}\right)$. As shown in Supplemental Figure 5, this resulted in significantly deeper light penetration for red stimuli (approximate exponential decay rate $\delta=1.84$ $\mathrm{mm})$ compared with blue stimuli $(\delta=0.588 \mathrm{~mm})$.

Additional calculations were performed to analyze how optical energy requirements for optogenetic defibrillation would be affected if red light were applied to the outer surface of the pericardium instead of directly to the epicardium. This illumination configuration was modeled by assuming the heart was surrounded by a layer of adipose tissue (i.e., epicardial fat) and a fibrous sac filled with fluid (i.e., visceral and parietal pericardial membranes plus pericardial fluid). Both layers were assumed to have uniform thickness based on nominal measurements from human patients: $1.7 \mathrm{~mm}$ for the pericardium (21) and $4.1 \mathrm{~mm}$ for the epicardial fat layer (22). To quantify how variability in the extent of epicardial fat would affect the light energy required for optogenetic defibrillation, we considered a range of possible adipose layer thicknesses ( 0 to $8 \mathrm{~mm}$ ) and calculated, for each case, the applied pericardial $E_{e}$ necessary to ensure that light with a particular $E_{e, 0}$ value was able to reach the epicardial surface based on the exponential decay approximation:
$E_{e}(x)=E_{e, 0} e^{-x / s}$

(Equation 3)

Optical decay rates $(\delta)$ for nonmyocardial layers were obtained from the available literature, with optical diffusivity values derived from absorption and scattering coefficients $\left(\mu_{\mathrm{a}}\right.$ and $\mu_{\mathrm{s}}^{\prime}$, respectively) using the following formulas:

$D=\frac{1}{3\left(\mu_{a}+\mu_{s}^{\prime}\right)}$

(Equation 4)

$\delta=\sqrt{\left(\frac{D}{\mu_{a}}\right)}$

(Equation 5)

For the epicardial fat layer, we used parameter values for red light (669 $\mathrm{nm})$ illumination of subcutaneous adipose tissue $\left(\mu_{\mathrm{a}}=0.0134 \mathrm{~mm}^{-1}\right.$ and $\left.\mu_{\mathrm{s}}^{\prime}=1.13 \mathrm{~mm}^{-1}, D=0.292 \mathrm{~mm}, \delta=4.66 \mathrm{~mm}\right)(60)$. Relevant measurements of the mammalian pericardium were not found in the literature; thus, for the pericardium in our model, we used optical parameters reported for the human peritoneum $\left(\mu_{\mathrm{a}}=0.0270 \mathrm{~mm}^{-1}, \mu_{\mathrm{s}}^{\prime}=1.83\right.$ $\left.\mathrm{mm}^{-1}, D=0.180 \mathrm{~mm}, \delta=2.56 \mathrm{~mm}\right)(61,62)$.

Protocol for initiation and optogenetic defibrillation of VT. Sustained arrhythmia ( $\geq 5$ seconds of self-sustained re-entrant activity) was initiated by a rapid pacing sequence resembling the clinical protocol for electrophysiological study in VT patients (59). A train of six 10-ms-long electrical stimuli (S1) was delivered to the ventricular apex at a cycle length of $350 \mathrm{~ms}$. This was followed by a premature stimulus (S2, coupling interval $=270 \mathrm{~ms}$ ), which induced sustained VT (Figure 4A, Supplemental Figure 3, and Supplemental Video 1). Three unique simulations were conducted with different optogenetic configurations: control (no illumination), blue illumination $(488 \mathrm{~nm})$, and red illumination $(669 \mathrm{~nm})$. For the latter 2 cases, light stimulation $\left(10 \mathrm{~mW} / \mathrm{mm}^{2}\right)$ was applied to the entire epicardial surface for 1 second. Light pulse onset time was at $t=4$ seconds, approximately 2 seconds after the delivery of the S2 stimulus.

Pseudo-ECGs. Extracellular potential from 2 points approximately $1 \mathrm{~cm}$ away from the left and right ventricular surfaces $\left(\varphi_{\mathrm{LV}}\right.$ and $\varphi_{\mathrm{RV}}$, respectively; black dots in Supplemental Figure 3) was calculated using an approach we have used in previous studies $(63,64)$. Pseudo-ECG signals (Figure 4, A and B, Supplemental Figure 6B, and Supplemental Video 2), analogous to clinical lead I (i.e., left arm minus right arm), were obtained by subtraction of $\varphi_{\mathrm{RV}}$ from $\varphi_{\mathrm{LV}^{*}}$

Analysis of diastolic depolarization and $\mathrm{Na}^{+}$current inactivation. To characterize minimal diastolic transmembrane voltage $\left(V_{m}\right)$ values within the free ventricular walls (without the septum) during illumination (Figure 5D), 3D maps of $V_{m}$ were output at high temporal resolution (1-ms time step) for the interval during which stimulation was applied ( $t=4-5$ seconds). Data within the first $100 \mathrm{~ms}$ (i.e., $t=4-4.1$ seconds) were excluded from analysis to ensure that these values were not biased toward either fast upstrokes associated with the initial response to optogenetic stimulation (i.e., directly light-induced action potentials) or low preillumination resting potentials. The threshold for 
$90 \%$ inactivation of $\mathrm{Na}^{+}$current (dashed line in Figure 5D) was derived by identification of the specific value $\left(V_{m}=-65.8 \mathrm{mV}\right)$ for which $h_{\infty}$ was equal to 0.1 in the human ventricular action potential model (43).

Statistics. Because of the exploratory nature of this study, effect sizes could not be predicted, and thus prior power analysis to determine the sample sizes could not be performed. Randomization and blinding for mouse strains were not applicable because the light pulse-induced stimulation is disclosing the ChR2 genotype. For statistical comparison (Figure 1, B-F, and Figure 2C), we considered only experiments in which at least 5 defibrillation attempts could be evaluated for each displayed parameter. For each mouse heart, the average termination rate for a given illumination parameter was calculated, displayed as 1 data point in the graphs, and used for subsequent statistical analysis as 1 biological replicate. Because of the non-normal distribution and the unequal variances of average arrhythmia termination rates, the following nonparametric tests were used: the Friedman test to estimate the influences of illumination parameters (Figure 1, D-F), the 1-way ANOVA Kruskal-Wallis with Dunn's multiple-comparison post-test for comparison between different mouse lines (Figure 1C), and the Wilcoxon matched pairs $t$ test for comparison between protocols with and without illumination in ChR2-expressing hearts (Figure 1B and Figure 2C). Statistics were calculated with GraphPad Prism (GraphPad Software). $P$ values less than 0.05 were considered statistically significant and are indicated by ${ }^{*} P \leq 0.05,{ }^{* *} P \leq 0.01$, and ${ }^{* * *} P \leq 0.001$ in the figures. Data are shown as mean \pm SEM unless otherwise indicated.

Study approval. All animal procedures were performed in accordance with the Guide for the Care and Use of Laboratory Animals pub- lished by the National Institutes of Health (8th edition, revised 2011) and were authorized by the local ethics review board (Landesamt für Natur, Umwelt und Verbraucherschutz Nordrhein-Westfalen, Germany, 84-02.04.2011.A292).

\section{Author contributions}

TB, PMB, BKF, NAT, and PS designed the study. TB, CCV, and PS performed the experiments in mice and analyzed the experimental data. HJA developed the human VT model. TVK and PMB performed and analyzed the simulations of the human VT model. TB, PMB, TVK, BKF, NAT, and PS wrote the manuscript.

\section{Acknowledgments}

We thank F. Holst for technical assistance and Penn Vector Core, Gene Therapy Program (University of Pennsylvania), for providing the AAV9-CAG-hChR2(H134R)-mCherry.WPRE.SV40 virus vector. This work was supported by the German Research Foundation (SA 1785/5-1, SA 1785/7-1 and Research Training Group 1873 to P. Sasse); the BONFOR Program, Medical Faculty, University of Bonn (O-162.0011 to P. Sasse); and the NIH (DP1 HL123271, R01 HL105216, R01 HL103428, and R01 HL126802 to N.A. Trayanova).

Address correspondence to: Philipp Sasse, Institute of Physiology I, University of Bonn, Life and Brain Center, Sigmund-FreudStrasse 25, 53127 Bonn, Germany. Phone: 49.228.6885.200; E-mail:philipp.sasse@uni-bonn.de.
1. Marcus GM, Chan DW, Redberg RF. Recollection of pain due to inappropriate versus appropriate implantable cardioverterdefibrillator shocks. Pacing Clin Electrophysiol. 2011;34(3):348-353.

2. Reynolds MR, et al. The frequency and incremental cost of major complications among medicare beneficiaries receiving implantable cardioverter-defibrillators. J Am Coll Cardiol. 2006;47(12):2493-2497.

3. Sohail MR, Henrikson CA, Braid-Forbes MJ, Forbes KF, Lerner DJ. Mortality and cost associated with cardiovascular implantable electronic device infections. Arch Intern Med. 2011;171(20):1821-1828.

4. Larsen GK, Evans J, Lambert WE, Chen Y, Raitt MH. Shocks burden and increased mortality in implantable cardioverter-defibrillator patients. Heart Rhythm. 2011;8(12):1881-1886.

5. Bruegmann T, et al. Optogenetic control of heart muscle in vitro and in vivo. Nat Methods. 2010;7(11):897-900.

6. Vogt CC, et al. Systemic gene transfer enables optogenetic pacing of mouse hearts. Cardiovasc Res. 2015;106(2):338-343.

7. Nussinovitch U, Gepstein L. Optogenetics for in vivo cardiac pacing and resynchronization therapies. Nat Biotechnol. 2015;33(7):750-754.

8. Arrenberg AB, Stainier DY, Baier H, Huisken J. Optogenetic control of cardiac function. Science. 2010;330(6006):971-974.

9. Zipes DP, Fischer J, King RM, Nicoll A deB, Jolly WW. Termination of ventricular fibrillation in dogs by depolarizing a critical amount of myocardium. Am J Cardiol. 1975;36(1):37-44.
10. Burton RA, et al. Optical control of excitation waves in cardiac tissue. Nat Photonics. 2015;9(12):813-816.

11. Bingen BO, et al. Light-induced termination of spiral wave arrhythmias by optogenetic engineering of atrial cardiomyocytes. Cardiovasc Res. 2014;104(1):194-205.

12. Maguire CT, Wakimoto H, Patel VV, Hammer PE, Gauvreau K, Berul CI. Implications of ventricular arrhythmia vulnerability during murine electrophysiology studies. Physiol Genomics. 2003;15(1):84-91.

13. Boyle PM, Williams JC, Ambrosi CM, Entcheva E, Trayanova NA. A comprehensive multiscale framework for simulating optogenetics in the heart. Nat Commun. 2013;4:2370.

14. Ambrosi CM, Boyle PM, Chen K, Trayanova NA, Entcheva E. Optogenetics-enabled assessment of viral gene and cell therapy for restoration of cardiac excitability. Sci Rep. 2015;5:17350.

15. Williams JC, et al. Computational optogenetics: empirically-derived voltage- and light-sensitive channelrhodopsin-2 model. PLoS Comput Biol. 2013;9(9):e1003220.

16. Karathanos TV, Bayer JD, Wang D, Boyle PM, Trayanova NA. Opsin spectral sensitivity determines the effectiveness of optogenetic termination of ventricular fibrillation in the human heart: a simulation study [published online ahead of print March 4, 2016]. JPhysiol. doi:10.1113/JP271739.

17. Klapoetke NC, et al. Independent optical excitation of distinct neural populations. Nat Methods. 2014;11(3):338-346.

18. Lin JY, Knutsen PM, Muller A, Kleinfeld D,
Tsien RY. ReaChR: a red-shifted variant of channelrhodopsin enables deep transcranial optogenetic excitation. Nat Neurosci. 2013;16(10):1499-1508.

19. Kleinlogel S, et al. Ultra light-sensitive and fast neuronal activation with the $\mathrm{Ca}^{2+}$-permeable channelrhodopsin CatCh. Nat Neurosci. 2011;14(4):513-518.

20. Bishop MJ, Rodriguez B, Eason J, Whiteley JP, Trayanova N, Gavaghan DJ. Synthesis of voltage-sensitive optical signals: application to panoramic optical mapping. Biophys J. 2006;90(8):2938-2945.

21. Sechtem U, Tscholakoff D, Higgins CB. MRI of the normal pericardium. AJR Am J Roentgenol. 1986;147(2):239-244.

22. Flüchter $S$, et al. Volumetric assessment of epicardial adipose tissue with cardiovascular magnetic resonance imaging. Obesity (Silver Spring). 2007;15(4):870-878.

23. Pezhouman A, et al. Molecular basis of hypokalemia-induced ventricular fibrillation. Circulation. 2015;132(16):1528-1537.

24. Glukhov AV, Flagg TP, Fedorov VV, Efimov IR, Nichols CG. Differential K(ATP) channel pharmacology in intact mouse heart. J Mol Cell Cardiol. 2010;48(1):152-160.

25. Billman GE. The cardiac sarcolemmal ATP-sensitive potassium channel as a novel target for anti-arrhythmic therapy. Pharmacol Ther. 2008;120(1):54-70.

26. Zipes DP, Wellens HJ. Sudden cardiac death. Circulation. 1998;98(21):2334-2351.

27. Arevalo HJ, et al. Arrhythmia risk stratification of patients after myocardial infarction using person- 
alized heart models. Nat Commun. 2016;7:11437.

28. Kim TI, et al. Injectable, cellular-scale optoelectronics with applications for wireless optogenetics. Science. 2013;340(6129):211-216.

29. Xu L, et al. 3D multifunctional integumentary membranes for spatiotemporal cardiac measurements and stimulation across the entire epicardium. Nat Commun. 2014;5:3329.

30. Zacchigna S, Zentilin L, Giacca M. Adeno-associated virus vectors as therapeutic and investigational tools in the cardiovascular system. Circ Res. 2014;114(11):1827-1846.

31. Werfel S, et al. Rapid and highly efficient inducible cardiac gene knockout in adult mice using AAV-mediated expression of Cre recombinase. Cardiovasc Res. 2014;104(1):15-23.

32. Nagel G, Brauner M, Liewald JF, Adeishvili N, Bamberg E, Gottschalk A. Light activation of channelrhodopsin-2 in excitable cells of Caenorhabditis elegans triggers rapid behavioral responses. Curr Biol. 2005;15(24):2279-2284.

33. Prakosa A, et al. Methodology for image-based reconstruction of ventricular geometry for patient-specific modeling of cardiac electrophysiology. Prog Biophys Mol Biol. 2014;115(2-3):226-234.

34. Deng D, et al. Accuracy of prediction of infarct-related arrhythmic circuits from imagebased models reconstructed from low and high resolution MRI. Front Physiol. 2015;6:282.

35. Ashikaga $\mathrm{H}$, et al. Feasibility of image-based simulation to estimate ablation target in human ventricular arrhythmia. Heart Rhythm. 2013;10(8):1109-1116.

36. Arevalo H, Plank G, Helm P, Halperin H, Trayanova N. Tachycardia in post-infarction hearts: insights from 3D image-based ventricular models. PLoS One. 2013;8(7):e68872.

37. Vadakkumpadan F, et al. Image-based models of cardiac structure with applications in arrhythmia and defibrillation studies. J Electrocardiol. 2009;42(2):157.e1-157.10.

38. Schmidt A, et al. Infarct tissue heterogeneity by magnetic resonance imaging identifies enhanced cardiac arrhythmia susceptibility in patients with left ventricular dysfunction. Circulation. 2007;115(15):2006-2014.

39. Raya SP, Udupa JK. Shape-based interpolation of multidimensional objects. IEEE Trans Med Imaging. 1990;9(1):32-42.

40. McDowell KS, et al. Methodology for patient-specific modeling of atrial fibrosis as a substrate for atrial fibrillation. J Electrocardiol. 2012;45(6):640-645.

41. Prassl AJ, et al. Automatically generated, ana- tomically accurate meshes for cardiac electrophysiology problems. IEEE Trans Biomed Eng. 2009;56(5):1318-1330.

42. Bayer JD, Blake RC, Plank G, Trayanova NA. A novel rule-based algorithm for assigning myocardial fiber orientation to computational heart models. Ann Biomed Eng. 2012;40(10):2243-2254.

43. ten Tusscher KH, Panfilov AV. Alternans and spiral breakup in a human ventricular tissue model. Am J Physiol Heart Circ Physiol. 2006;291(3):H1088-H1100.

44. Pu J, Boyden PA. Alterations of $\mathrm{Na}^{+}$currents in myocytes from epicardial border zone of the infarcted heart. A possible ionic mechanism for reduced excitability and postrepolarization refractoriness. Circ Res. 1997;81(1):110-119.

45. Dun W, Baba S, Yagi T, Boyden PA. Dynamic remodeling of $\mathrm{K}^{+}$and $\mathrm{Ca}^{2+}$ currents in cells that survived in the epicardial border zone of canine healed infarcted heart. Am J Physiol Heart Circ Physiol. 2004;287(3):H1046-H1054.

46. Jiang M, Cabo C, Yao J, Boyden PA, Tseng G. Delayed rectifier K currents have reduced amplitudes and altered kinetics in myocytes from infarcted canine ventricle. Cardiovasc Res. 2000;48(1):34-43.

47. Decker KF, Rudy Y. Ionic mechanisms of electrophysiological heterogeneity and conduction block in the infarct border zone. Am J Physiol Heart Circ Physiol. 2010;299(5):H1588-H1597.

48. Cabo C, Boyden PA. Electrical remodeling of the epicardial border zone in the canine infarcted heart: a computational analysis. Am J Physiol Heart Circ Physiol. 2003;284(1):H372-H384.

49. Taggart $P$, et al. Inhomogeneous transmural conduction during early ischaemia in patients with coronary artery disease. J Mol Cell Cardiol. 2000;32(4):621-630.

50. Glukhov AV, et al. Transmural dispersion of repolarization in failing and nonfailing human ventricle. Circ Res. 2010;106(5):981-991.

51. Yao JA, Hussain W, Patel P, Peters NS, Boyden PA, Wit AL. Remodeling of gap junctional channel function in epicardial border zone of healing canine infarcts. Circ Res. 2003;92(4):437-443.

52. Vigmond EJ, Weber dos Santos R, Prassl AJ, Deo M, Plank G. Solvers for the cardiac bidomain equations. Prog Biophys Mol Biol. 2008;96(1-3):3-18.

53. Vigmond EJ, Hughes M, Plank G, Leon LJ. Computational tools for modeling electrical activity in cardiac tissue. J Electrocardiol. 2003;36(suppl):69-74.

54. Rodríguez B, Li L, Eason JC, Efimov IR, Trayanova
NA. Differences between left and right ventricular chamber geometry affect cardiac vulnerability to electric shocks. Circ Res. 2005;97(2):168-175

55. Bishop MJ, Rodriguez B, Qu F, Efimov IR, Gavaghan DJ, Trayanova NA. The role of photon scattering in optical signal distortion during arrhythmia and defibrillation. Biophys J. 2007;93(10):3714-3726.

56. Rantner LJ, Arevalo HJ, Constantino JL, Efimov IR, Plank G, Trayanova NA. Three-dimensional mechanisms of increased vulnerability to electric shocks in myocardial infarction: altered virtual electrode polarizations and conduction delay in the peri-infarct zone. J Physiol (Lond). 2012;590(18):4537-4551.

57. Boyle PM, Karathanos TV, Entcheva E, Trayanova NA. Computational modeling of cardiac optogenetics: Methodology overview \& review of findings from simulations. Comput Biol Med. 2015;65:200-208.

58. Mattis J, et al. Principles for applying optogenetic tools derived from direct comparative analysis of microbial opsins. Nat Methods. 2012;9(2):159-172.

59. Wellens HJ, Brugada P, Stevenson WG. Programmed electrical stimulation of the heart in patients with life-threatening ventricular arrhythmias: what is the significance of induced arrhythmias and what is the correct stimulation protocol? Circulation. 1985;72(1):1-7.

60. Bashkatov AN, Genina ÉA, Kochubey VI, Tuchin VV. Optical properties of the subcutaneous adipose tissue in the spectral range $400-2500 \mathrm{~nm}$. Opt Spectrosc. 2005;99(5):836-842.

61. Sandell JL, Zhu TC. A review of in-vivo optical properties of human tissues and its impact on PDT. J Biophotonics. 2011;4(11-12):773-787.

62. Wang HW, et al. Broadband reflectance measurements of light penetration, blood oxygenation, hemoglobin concentration, and drug concentration in human intraperitoneal tissues before and after photodynamic therapy. J Biomed Opt 2005;10(1):14004

63. Boyle PM, Veenhuyzen GD, Vigmond EJ. Fusion during entrainment of orthodromic reciprocating tachycardia is enhanced for basal pacing sites but diminished when pacing near Purkinje system end points. Heart Rhythm. 2013;10(3):444-451.

64. Boyle PM, Massé S, Nanthakumar K, Vigmond EJ. Transmural IK(ATP) heterogeneity as a determinant of activation rate gradient during early ventricular fibrillation: mechanistic insights from rabbit ventricular models. Heart Rhythm. 2013;10(11):1710-1717. 